\title{
Age and Gender Determination from Finger Prints using RVA and det Coefficients
}

\author{
Ravi Wadhwa, Maninder Kaur, Dr. K.V.P. Singh \\ ${ }^{I}$ Doaba Institute of Eng. and Tech., Kharar, Mohali \\ ${ }^{2}$ DIET, Kharar, Mohali ${ }^{3}$ DIET, Kharar, Mohali
}

\begin{abstract}
In the presented work, age and gender of a person from finger print impression has been worked out. The novelty in the solution lies in the fact that the identification of age and sex is independent from the pressure i.e. finger prints thickness or ridge/valley thickness. The age and gender finger prints are classified on the basis of ridge to valley area, entropy and rms value of dct coefficients. The classification is described in the result section
\end{abstract}

Keywords: - DPI $\rightarrow$ Dots per Inch, RVA $\rightarrow$ Ridge to valley Area, RMS $\rightarrow$ Root Mean Square

\section{INTRODUCTION}

Sex identification of suspect from crime scene is an important task in forensic science that minimizes the search population of suspects. Existing methods for gender classification have limited use for crime scene investigation because they depend on the availability of teeth, bones, or other identifiable body parts having physical features that allow gender and age estimation by conventional methods. Various methodologies has been used to identify the gender using different biometrics traits such as face, gait, iris, hand shape, speech and fingerprint. Fingerprint has been used as a biometric for the gender and age identification because of its unique nature and do not change throughout the life of an individual.

Wavelet transform is a popular tool in image processing and computer vision because of its complete theoretical framework, the great flexibility for choosing bases and the low computational complexity. As wavelet features has been popularized by the research community for wide range of applications including fingerprint recognition, face recognition and gender identification using face, authors have confirmed the efficiency of the DWT approach for the gender identification using fingerprint. The SVD approach is selected for the gender discrimination because of its good information packing characteristics and potential strengths in demonstrating results. The SVD method is considered as an information oriented technique since it uses principal components analysis procedures (PCA), a form of factor analysis, to concentrate information before examining the primary analytic issues of interest. K-nearest neighbors (KNN), gives very strong consistent results.

\section{RELATED WORKS}

A novel method of gender Classification from fingerprint is proposed based on discrete wavelet transform (DWT) and singular value decomposition (SVD). The classification is achieved by extracting the energy computed from all the sub-bands of DWT combined with the spatial features of non-zero singular values obtained from the SVD of fingerprint images. K nearest neighbor (KNN) used as a classifier. This method is experimented with the internal database of 3570 fingerprints finger prints in which 1980 were male fingerprints and 1590 were female fingerprints. Finger wise gender classification is achieved which is $94.32 \%$ for the left hand little fingers of female persons and $95.46 \%$ for the left hand index finger of male persons. Gender classification for any finger of male persons tested is attained as $91.67 \%$ and $84.69 \%$ for female persons respectively. Overall classification rate is $88.28 \%$ has been achieved [1].

This study evaluated fingerprint quality across two populations, elderly and young, in order to assess age and moisture as potential factors affecting utility image quality. Specifically, the examination of these variables was conducted on a population over the age of 62, and a population between the ages of 18 and 25, using two fingerprint recognition devices (capacitance and optical). Collected individual variables included: age, gender, ethnic background, handedness, moisture content of each index finger, occupation(s), subject's use of hand moisturizer, and prior usage of fingerprint devices. Computed performance measures included failure to enroll, and quality scores. The results indicated there was statistically significant evidence that both age and moisture affected effectiveness image quality of each index finger at $a=0.01$ on the optical device, and there was statistically significant evidence that age affected effectiveness image quality of each index finger on the capacitance device, but moisture was only significant for the right index finger at $a=0.01[2]$. 
Fingerprint evidence is undoubtedly the most reliable and acceptable evidence till date in the court of law. Due to the immense potential of fingerprints as an effective method of identification an attempt has been made in the present work to analyze their correlation with gender of an individual. This prospective study was carried out over a period of 2 months among 500 public people( 250 male \& 250 female) belonging to the various age groups between $1-90$. Features extracted were; ridge count, ridge thickness to valley thickness ratio (RTVTR), white lines count, and ridge count asymmetry, and pattern type concordance. For age classification Neural Network (NN) was used for the classification using the most dominant features. Results are calculated by our proposed method and are compared with the existing methods like KNN and LVQ. This analysis makes the proposed method better accurate than existing methods [3].

Gender classification from fingerprints is an important step in forensic anthropology in order to identify the gender of a criminal and minimize the list of suspects search. A dataset of 10-fingerprint images for 2200 persons of different ages and gender (1100 males and 1100 females) was analyzed. Features extracted were; ridge count, ridge thickness to valley thickness ratio (RTVTR), white lines count, ridge count asymmetry, and pattern type concordance. Fuzzy CMeans (FCM), Linear Discriminant Analysis (LDA), and Neural Network (NN) were used for the classification using the most dominant features. We obtained results of $80.39 \%, 86.5 \%$, and $88.5 \%$ using FCM, LDA, and NN, respectively. Results of this analysis make this method a prime candidate to utilize In forensic anthropology for gender classification in order to minimize the suspects search list by getting a likelihood value for the criminal gender [4].

This study was conducted with an aim to establish a relationship between sex and fingerprint ridge density. The fingerprints were taken from 500 subjects ( 250 males and 250 females) in the age group of 18-60 years. After taking fingerprints, the ridges were counted in the upper portion of the radial border of each print for all ten fingers and mean value was calculated. The results have shown that a finger print ridge of $<13$ ridges $/ 25 \mathrm{~mm} 2$ is more likely of male origin and finger print ridge of $>14$ ridges $/ 25 \mathrm{~mm} 2$ is more likely of female origin. It has been successful to support the hypothesis that women tend to have a statistically significant greater ridge density than men [5].

The forensic investigators always search for fingerprint evidence which is seen as one of the best types of physical evidence linking a suspect to the crime. In this paper discrete wavelet transform (DWT) and the singular value decomposition (SVD) has been used to estimate a person's age using his/her fingerprint. The most robust $\mathrm{K}$ nearest neighbor (KNN) used as a classifier. The evaluation of the system is carried on using internal database of 3570 fingerprints in which 1980 were male fingerprints and 1590 were female fingerprints. Tested fingerprint is grouped into any one of the following five groups: up to 12, 13-19, 20-25, 26-35 and 36 and above. By the proposed method, fingerprints were classified accurately by $96.67 \%, 71.75 \%, 86.26 \%$, $76.39 \%$ and $53.14 \%$ in five groups respectively for male and by $66.67 \%, 63.64 \%, 76.77 \%, 72.41 \%$ and $16.79 \%$ for female. Finger-wise and Hand-wise results of age estimation also achieved [6].

Fingerprint recognition belongs to one of the most often used biometric technologies worldwide. It is believed that fingerprints could be used for the recognition of a person in nearly any case; however there exist many cases, where the fingerprint recognition could not be used. There exist some influencing factors [1] that have an impact to the process of fingerprint recognition, e.g. the environmental influences, dirtiness on finger or the sensor, electromagnetic radiation or diseases. This chapter deals with the circumstances which

influence the quality of fingerprints - we are limited on skin diseases here, further we explain how we can evaluate the quality of the acquired fingerprint [7].

This article proposes several improvements to an adaptive fingerprint enhancement method that is based on contextual filtering. The term adaptive implies that parameters of the method are automatically adjusted based on the input fingerprint image. Five processing blocks comprise the adaptive fingerprint enhancement method, where four of these blocks are updated in our proposed system. Hence, the proposed overall system is novel. The four updated processing blocks are; preprocessing, global analysis, local analysis and matched filtering. In the pre-processing and local analysis blocks, a nonlinear dynamic range adjustment method is used. In the global analysis and matched filtering blocks, different forms of order statistical filters are applied. These processing blocks yield an improved and new adaptive fingerprint image processing method. The performance of the updated processing blocks is presented in the evaluation part of this paper. The algorithm is evaluated towards the NIST developed NBIS software for fingerprint recognition on FVC databases [8].

\section{Methodology}

The presented work has been divided into four sections namely: [a] Image Acquisition and Preprocessing, [b] Ridge to Valley Area Computation, [c] Entropy Computation

\section{Image AcQuisition ANd Preprocessing}

The finger prints are acquired by using a finger print scanner. In the presented work, the finger prints are collected using the Futronic make finger print scanner of 300 dpi resolution in jpeg format. The scanned 
finger prints are converted to gray scale images using the rgb2gray command in Matlab. Further, the images are binarized using the Otsu algorithm. In case of Otsu algorithm, the weighted sum of within-class variances of the foreground and background pixels is minimized to establish an optimum threshold. Recall that minimization of within class variances is tantamount to the maximization of between-class scatter. This method gives satisfactory results when the numbers of pixels in each class are close to each other.

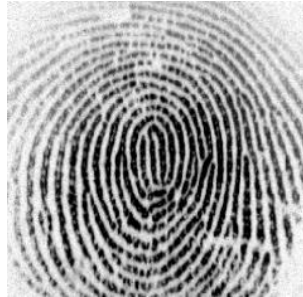

Fig. 1

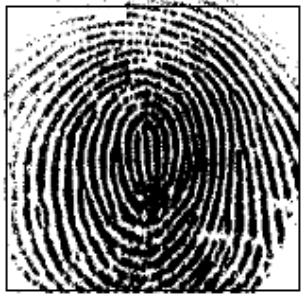

Fig. 2

Original Image Image after preprocessing and bounded box

\section{Ridge to valley Area (RVA) Computation}

The binarized finger print image is bounded in a bounded box in confine the finger prints in a closed circumference. Excess area is removed or not considered in calculation. Fig. 2 shows the finger print image after preprocessing and bounded in a bounded box.

Ridges are defined as the black impression in the finger print images while vallies are that of white portion in the same. Ridge and valley areas in finger print image may be defined as the total no. of black and white pixels respectively within the bounding box and is given by:

Say, $\quad A_{r}=$ Total no. of Black Pixels,

and $\quad \mathrm{A}_{\mathrm{v}}=$ Total no. of White Pixels

Where $A_{b}$ and $A_{w}$ are the areas of ridges and vallies. Ridge to valley area (RVA) is given by:

$$
\mathrm{RVA}=\mathrm{A}_{\mathrm{r}} / \mathrm{A}_{\mathrm{v}}
$$

\section{ENTROPY COMPUTATION WITHIN BOUNDING BOX}

The expression of the information entropy of an image is given by:

$$
H=-\sum_{i=0}^{L-1} p_{i} \ln p_{i}
$$

Where $\mathrm{L}$ denotes the number of gray level, $\mathrm{P}_{\mathrm{i}}$ equals the ratio between the number of pixels whose gray value equals I ( 0 to L-1) and the total pixel number contained in an image. The inormation entropy measures the richness of information in an image. If $\mathrm{P}_{\mathrm{i}}$ is the constant for an arbitrary gray level, then the entropy is maximum.

\section{RMS Value of DCT CoefFicients}

The definition of the two-dimensional DCT for an input image A and output image B is given by:

$B_{p q}=\alpha_{p} \alpha_{q} \sum_{m=0}^{M-1} \sum_{n=0}^{N-1} A_{m n} \cos \frac{\pi(2 m+1) p}{2 M} \cos \frac{\pi(2 n+1) q}{2 N}, \quad \begin{aligned} & 0 \leq p \leq M-1 \\ & 0 \leq q \leq N-1\end{aligned}$

$\alpha_{p}=\left\{\begin{array}{ll}1 / \sqrt{M}, & p=0 \\ \sqrt{2 / M}, & 1 \leq p \leq M-1\end{array} \quad \alpha_{q}= \begin{cases}1 / \sqrt{N}, & q=0 \\ \sqrt{2 / N}, & 1 \leq q \leq N-1\end{cases}\right.$

where $\mathrm{M}$ and $\mathrm{N}$ are the row and column size of $\mathrm{A}$, respectively.

The image is divided into blocks of $8 \times 8$. The dct coefficients of $8 \times 8$ image block are computed using the above equations. For an $8 \times 8$ image pixel block, we get an $8 \times 8$ dct coefficients matrix. And therefore, in total 8 diagonal dct coefficients. For a 96x96 pixels image, we get 144 blocks of 8x8 pixels, thereby giving 1152 $(=144 \times 8)$ no. of diagonal det coefficients. The RMS value of diagonal det coefficients is given by: 


$$
\operatorname{dct}_{\mathrm{rms}}=\sqrt{\frac{\sum_{\mathrm{i}=1}^{\mathrm{N}} \mathrm{det}_{\mathrm{i}}^{2}}{\mathrm{~N}}}
$$

Where dct $_{\mathrm{i}}$ is the $\mathrm{i}_{\mathrm{th}}$ dct coefficient.

\section{RESULTS}

The computed value of entropy RVA and dct $_{\mathrm{rms}}$ for finger print images are tabulated in the table below. For gender classification, a data base of 100 finger prints from male and female of the same age group is used. The results for some of the finger print data are tabulated in the table no. 1 and 2.

\section{CONCLUSION}

The results are compiled using 500 dpi finger print scanner of Futronic Make. The trend as observed from the table mixed to some extent but can be resolved distinctly by generating more data male and female candidates and finally can be generalized to differentiate the gender based on finger print. Further, it has been observed that the age groups show a constant variation from one age group to net age group. The result tables show the trends and can be analyzed.

\section{REFERENCES}

[1] Gnanasivam P1, and Dr. Muttan S2, "Fingerprint Gender Classification using Wavelet Transform and Singular Value Decomposition “, IEEE-2012

[2] Nathan C. Sickler \& Stephen J Elliott, PhD, "AN EVALUATION OF FINGERPRINT IMAGE QUALITY ACROSS AN ELDERLY POPULATION VIS-A-VIS AN 18-25 YEAR OLD POPULATION", IEEE-2005

[3] S. Sudha Ponnarasi, M. Rajaram, "Age Classification System Anchored in Fingerprint Minutiae Extraction”, European Journal of Scientific Research,ISSN 1450-216X Vol.73 No.2 (2012),

[4] Ahmed Badawi1, Mohamed Mahfouz1, Rimon Tadross1, Richard Jantz2, "Fingerprint-Based Gender Classification", IEEE-2005

[5] Dr. Sudesh Gungadin, "SEX DETERMINATION FROM FINGERPRINT RIDGE DENSITY", Internet Journal of Medical Update 2007

[6] P. Gnanasivam \& Dr. S. Muttan, "Estimation of Age Through Fingerprints Using Wavelet Transform and Singular Value Decomposition", International Journal of Biometrics and Bioinformatics (IJBB), Volume (6) : Issue (2) : 2012, pp 58

[7] Michal Dolezel, Martin Drahansky, Jaroslav Urbanek, Eva Brezinova and Tai-hoon Kim, "Influence of Skin Diseases on Fingerprint Quality and Recognition", INTECH

[8] Josef Str”om Bart ${ }^{\circ}$ un ek1, Student Member, IEEE, Mikael Nilsson2, Member, IEEE, Benny S“allberg1, Member, IEEE, and Ingvar Claesson1, Member, IEEE, "Adaptive Fingerprint Image Enhancement with Emphasis on Pre-processing of Data”, IEEE-2011

\section{Author Profile}

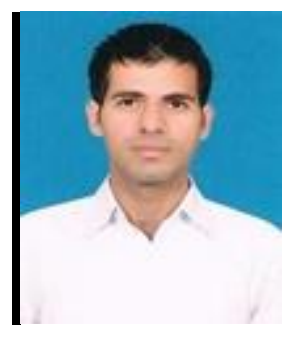

Mr. Ravi Wadhwa ${ }^{1}$ is pursuing M.Tech. in Electronics and Communication Engineering from DIET, Kharar. His current field of working is in bio-medical imaging system development and research field. 
Age and Gender Determination from Finger Prints using RVA and dct Coefficients

\begin{tabular}{|c|c|c|c|c|c|c|c|c|c|c|c|c|c|}
\hline $\begin{array}{c}\text { image } \\
\text { no. }\end{array}$ & age & M/F & \multicolumn{10}{|c|}{ RIDGE TO VELLY AREA(RVA } & AVG. \\
\hline 1 & 22 & F & 0.228 & 0.231 & 0.228 & 0.228 & 0.231 & 0.23 & 0.222 & 0.231 & 0.231 & 0.23 & 0.229 \\
\hline 2 & 25 & F & 0.229 & 0.23 & 0.229 & 0.23 & 0.229 & 0.229 & 0.23 & 0.232 & 0.231 & 0.232 & 0.23 \\
\hline 3 & 30 & F & 0.228 & 0.232 & 0.229 & 0.232 & 0.233 & 0.231 & 0.235 & 0.233 & 0.231 & 0.23 & 0.231 \\
\hline 4 & 25 & F & 0.229 & 0.234 & 0.232 & 0.232 & 0.23 & 0.234 & 0.228 & 0.225 & 0.229 & 0.232 & 0.231 \\
\hline 5 & 28 & F & 0.231 & 0.23 & 0.232 & 0.228 & 0.231 & 0.23 & 0.231 & 0.23 & 0.232 & 0.229 & 0.23 \\
\hline 6 & 18 & F & 0.235 & 0.234 & 0.233 & 0.233 & 0.233 & 0.232 & 0.228 & 0.231 & 0.231 & 0.229 & 0.232 \\
\hline 7 & 20 & F & 0.234 & 0.227 & 0.229 & 0.23 & 0.232 & 0.234 & 0.234 & 0.229 & 0.228 & 0.228 & 0.231 \\
\hline 8 & 23 & F & 0.231 & 0.231 & 0.23 & 0.232 & 0.23 & 0.23 & 0.231 & 0.229 & 0.231 & 0.233 & 0.231 \\
\hline 9 & 21 & F & 0.229 & 0.227 & 0.233 & 0.231 & 0.231 & 0.231 & 0.233 & 0.233 & 0.233 & 0.229 & 0.231 \\
\hline 10 & 18 & F & 0.23 & 0.231 & 0.228 & 0.232 & 0.231 & 0.231 & 0.23 & 0.228 & 0.232 & 0.23 & 0.23 \\
\hline 11 & 23 & M & 0.24 & 0.232 & 0.229 & 0.229 & 0.228 & 0.237 & 0.226 & 0.232 & 0.23 & 0.233 & 0.232 \\
\hline 12 & 28 & M & 0.232 & 0.228 & 0.228 & 0.227 & 0.226 & 0.237 & 0.235 & 0.227 & 0.23 & 0.23 & 0.23 \\
\hline 13 & 25 & M & 0.231 & 0.228 & 0.236 & 0.233 & 0.23 & 0.228 & 0.235 & 0.235 & 0.234 & 0.231 & 0.232 \\
\hline 14 & 28 & M & 0.231 & 0.227 & 0.231 & 0.232 & 0.233 & 0.234 & 0.23 & 0.233 & 0.229 & 0.229 & 0.231 \\
\hline 15 & 19 & M & 0.231 & 0.234 & 0.229 & 0.231 & 0.228 & 0.232 & 0.23 & 0.233 & 0.231 & 0.229 & 0.231 \\
\hline 16 & 20 & M & 0.235 & 0.234 & 0.228 & 0.229 & 0.23 & 0.235 & 0.232 & 0.228 & 0.231 & 0.225 & 0.231 \\
\hline 17 & 20 & M & 0.236 & 0.234 & 0.227 & 0.232 & 0.231 & 0.233 & 0.232 & 0.231 & 0.229 & 0.231 & 0.232 \\
\hline 18 & 26 & M & 0.232 & 0.231 & 0.23 & 0.231 & 0.231 & 0.228 & 0.232 & 0.229 & 0.232 & 0.232 & 0.231 \\
\hline 19 & 24 & M & 0.233 & 0.227 & 0.232 & 0.23 & 0.234 & 0.234 & 0.227 & 0.231 & 0.231 & 0.228 & 0.231 \\
\hline 20 & 23 & M & 0.229 & 0.23 & 0.23 & 0.231 & 0.231 & 0.234 & 0.232 & 0.231 & 0.228 & 0.231 & 0.231 \\
\hline
\end{tabular}

OVERALL RESULT

\begin{tabular}{|c|c|c|c|c|c|}
\hline image no. & age & $\mathrm{m} / \mathrm{f}$ & ENTROPY & RVA & SD \\
\hline 1 & 22 & $\mathrm{~F}$ & 5.96218 & 0.229 & 12.0315 \\
\hline 2 & 25 & $\mathrm{~F}$ & 5.9155 & 0.2301 & 5.4329 \\
\hline 3 & 30 & $\mathrm{~F}$ & 6.0461 & 0.2314 & 12.881 \\
\hline 4 & 25 & $\mathrm{~F}$ & 5.7615 & 0.2305 & 11.4854 \\
\hline 5 & 28 & $\mathrm{~F}$ & 5.91521 & 0.2304 & 9.0771 \\
\hline 6 & 18 & $\mathrm{~F}$ & 6.03356 & 0.2319 & 10.9596 \\
\hline 7 & 20 & $\mathrm{~F}$ & 6.17579 & 0.2305 & 10.6758 \\
\hline 8 & 23 & $\mathrm{~F}$ & 5.96513 & 0.2308 & 5.33 \\
\hline 9 & 21 & $\mathrm{~F}$ & 5.8888 & 0.231 & 9.6877 \\
\hline 10 & 18 & $\mathrm{~F}$ & 5.80462 & 0.2303 & 6.6864 \\
\hline 11 & 23 & $\mathrm{M}$ & 6.00865 & 0.2316 & 18.7529 \\
\hline 12 & 28 & $\mathrm{M}$ & 6.3454 & 0.23 & 11.1234 \\
\hline 13 & 25 & $\mathrm{M}$ & 6.3869 & 0.2321 & 10.0414 \\
\hline 14 & 28 & $\mathrm{M}$ & 6.2883 & 0.2309 & 12.4652 \\
\hline 15 & 19 & $\mathrm{M}$ & 6.11717 & 0.2308 & 13.4545 \\
\hline 16 & 20 & $\mathrm{M}$ & 6.38188 & 0.2307 & 11.4949 \\
\hline 17 & 20 & $\mathrm{M}$ & 5.94997 & 0.2316 & 11.6299 \\
\hline 18 & 26 & $\mathrm{M}$ & 6.14755 & 0.2308 & 11.9525 \\
\hline 19 & 24 & $\mathrm{M}$ & 6.71808 & 0.2307 & 17.3516 \\
\hline 20 & 23 & $\mathrm{M}$ & 6.11321 & 0.2307 & 9.9082 \\
\hline
\end{tabular}

